\title{
Peer
}

\section{Translating the hemodynamic response: why focused interdisciplinary integration should matter for the future of functional neuroimaging}

\author{
Sigita Cinciute \\ Institute of Biosciences, Life Sciences Center, Vilnius University, Vilnius, Lithuania
}

\section{ABSTRACT}

The amount of information acquired with functional neuroimaging techniques, particularly fNIRS and fMRI, is rapidly growing and has enormous potential for studying human brain functioning. Therefore, many scientists focus on solving computational neuroimaging and Big Data issues to advance the discipline. However, the main obstacle-the accurate translation of the hemodynamic response (HR) by the investigation of a physiological phenomenon called neurovascular couplingis still not fully overcome and, more importantly, often overlooked in this context. This article provides a brief and critical overview of significant findings from cellular biology and in vivo brain physiology with a focus on advancing existing HR modelling paradigms. A brief historical timeline of these disciplines of neuroscience is presented for readers to grasp the concept better, and some possible solutions for further scientific discussion are provided.

Submitted 11 October 2018 Accepted 14 February 2019 Published 25 March 2019

Corresponding author Sigita Cinciute,

sigita.cinciute@gmail.com

Academic editor

Jafri Abdullah

Additional Information and Declarations can be found on page 15

DOI 10.7717/peerj.6621

(c) Copyright

2019 Cinciute

Distributed under

Creative Commons CC-BY 4.0

OPEN ACCESS
Subjects Biophysics, Cell Biology, Molecular Biology, Neuroscience

Keywords Cerebrovascular regulation, Healthcare, Hemodynamic response, Neuroscience, Brain, Computational modelling, Functional near-infrared spectroscopy, Neurovascular coupling, Functional magnetic resonance imaging

\section{INTRODUCTION}

Modern functional neuroimaging methods cover broad spatial and temporal scales (Pouratian et al., 2003) and facilitate the important exploration of the functional organisation of the human brain in health and disease (Liu et al., 2015). Numerous statistical or methodological challenges are addressed with this complexity. However, some threats arise from fundamental conceptual challenges that remain widely underappreciated within the clinical and neuroimaging communities (Poldrack \& Yarkoni, 2016). Current trends in neuroimaging and computational neuroscience promote the advanced mathematical modelling of human brain function based on neuroimaging, and the implications associated with the use of Big Data (Hansen et al., 2014) in scientific research and healthcare innovations. These multidisciplinary interactions between different branches of science are vital for overall scientific progress. However, some main conceptual challenges may remain shadowed by massive trends and become a barrier to progress. For example, the ability to assess neural activity in a non-invasive way by 
measuring the brain's circulation of blood has revolutionised neuroscience. As a result, we are witnesses to enormous growth in the field of human brain research (Raichle, 2009; Toga, 2015). Each of the functional neuroimaging techniques used today, such as functional near-infrared spectroscopy (fNIRS), functional magnetic resonance imaging (fMRI), positron emission tomography (PET) or single photon emission computed tomography (SPECT) explore different metabolic or particular physiological events, but all are based on the physiological principles of neurovascular coupling (NVC). NVC is the process by which active brain regions induce a local increase in blood flow to match their energy demands via the dilation of capillaries and arterioles through various cellular signalling paths (Mishra et al., 2016). Capillary dilation generates a significant portion of the blood flow increase, evoked by neuronal activity (Hall et al., 2014), and is expected to contribute substantially to the observed hemodynamic response (HR) (Lindauer et al., 2010). Nevertheless, our understanding of NVC in humans despite its importance is still incomplete due to a lack of appropriate and consistent analysis strategies and stimulation paradigms (Hillman, 2014; Phillips et al., 2016). Despite their technological differences, widely used functional neuroimaging techniques, such as fMRI and less known but prominent fNIRS, are mainly based on the common underlying phenomenon of NVC (Hillman, 2014; Huneau, Benali \& Chabriat, 2015; Iadecola, 2017; Phillips et al., 2016). It makes some results of current experimental methods ambiguous compared to the more in-depth fundamental perspective (Lindauer et al., 2010; Phillips et al., 2016; Sotero \& Trujillo-Barreto, 2007).

The scientific knowledge is always limited to some extent and arguably with each new finding. However, the real concerns occur summarising that at the moment, a big part of scientific and clinical research production is based on sophisticated mathematical manipulations of neuroimaging data which was derived from the observations of NVC, referred to as a HR. A generalisation like this emphasises the need of tighter and focused interdisciplinary integration within particular neuroscience fields to improve the translation of physiological signal into neuroimaging data, which later is processed with sophisticated mathematical and statistical methods. The goal of this article was to fill the gap between the critical and brief overviews of one of the under-appreciated neuroimaging challenge: accurate translation of the HR to scientific and clinical findings. The next objective was to provide the scientific reader with a summary of key aspects and analyse why this problem is relevant to those that are interested in or directly involved in human cognition and behaviour neuroscience or clinical research. To serve this purpose, this article conceptualises the current knowledge of NVC from several perspectives, mainly cellular and molecular neuroscience (CMN), and functional neuroimaging (particularly fNIRS and fMRI as they are closely related).

\section{SURVEY METHODOLOGY}

The review was designed with a focus on the existing scientific paradigm in human brain functional neuroimaging research, mainly addressing the absence of necessary interactions between different multidisciplinary branches of neuroscience such as cellular biology, human brain physiology and computational modelling (CM). The articles that were 
reviewed in this paper were identified in databases (e.g. Google Scholar, PubMed, ScienceDirect) and subject-specific professional journals and websites (e.g. PLOS, PeerJ, Frontiers, Journal of Cerebral Blood Flow \& Metabolism). The literature review was assured to be unbiased and comprehensive by narrowing down the exploration by searching for original research articles and reviews that discuss (i) cerebrovascular regulation; functional hyperaemia; NVC; astroglial network; the origin of the HR signal in fNIRS; the origin of the blood-oxygen-level-dependent (BOLD) signal in fMRI; or (ii) the biophysical model of fNIRS and fMRI signals; the CM methods used in fNIRS and fMRI; and (iii) studies that compare both methods or combine them in humans. The author also searched for articles in cellular and molecular biology, animal studies, subsequently regarding studies in health and disease. However, it was done only in combination with the previously mentioned search criteria to identify relevant publications. The other inclusion criteria for selected articles required that articles would be directly related to the topic and would not exhaustively cover unrelated material such as other neuroscience methods if the results were not directly comparable with functional neuroimaging.

\section{WHAT IS A HEMODYNAMIC RESPONSE?}

The human brain represents only $2 \%$ of the total body mass. About $25 \%$ of the oxygen and from $20 \%$ to $70 \%$ of the glucose consumed by the human body is dedicated to cerebral functions (Herculano-Houzel, 2011). The maintenance and restoration of the ion gradients dissipated by signalling processes such as post-synaptic and action potentials, as well as the uptake and recycling of neurotransmitters are the primary processes contributing to the high brain's energy needs (Attwell et al., 2010). The brain blood circulation system actively regulates the constant demand and supply. However, active brain regions are often provided more than they require. One of the researchers poetically illustrated it as 'watering the garden for the sake of a single thirsty flower' (Malonek \& Grinvald, 1996). This overcompensation or functional hyperaemia is a fundamental phenomenon in normal brain function. It was first confirmed by (Roy \& Sherrington, 1890) and defines the dilation of arterioles and capillaries of a brain region in response to a local episode of high neuronal activity. Functional hyperaemia is a generalised term for the outcome of a complex cerebrovascular regulation mechanism which will be briefly discussed in this section. At this point, the term HR is associated with the quantitative measures of functional hyperaemia using fNIRS and fMRI. In fMRI, it is better known as the HR function (HRF) to imply its mathematical properties. Further, in the text, only the HR term will be used, as it describes an observation of a physiological NVC event common for both techniques.

Functional magnetic resonance imaging and fNIRS have different capacities to explore human brain functions. As was introduced, both methods, despite their data acquisition differences, are based on a common underlying phenomenon termed NVC. However, fMRI is more common in general, due to its broad application possibilities and historical background, especially in clinical practice (Glover, 2011), while ANIRS was primarily used for the bedside monitoring of infants, and other fields where fMRI were not 

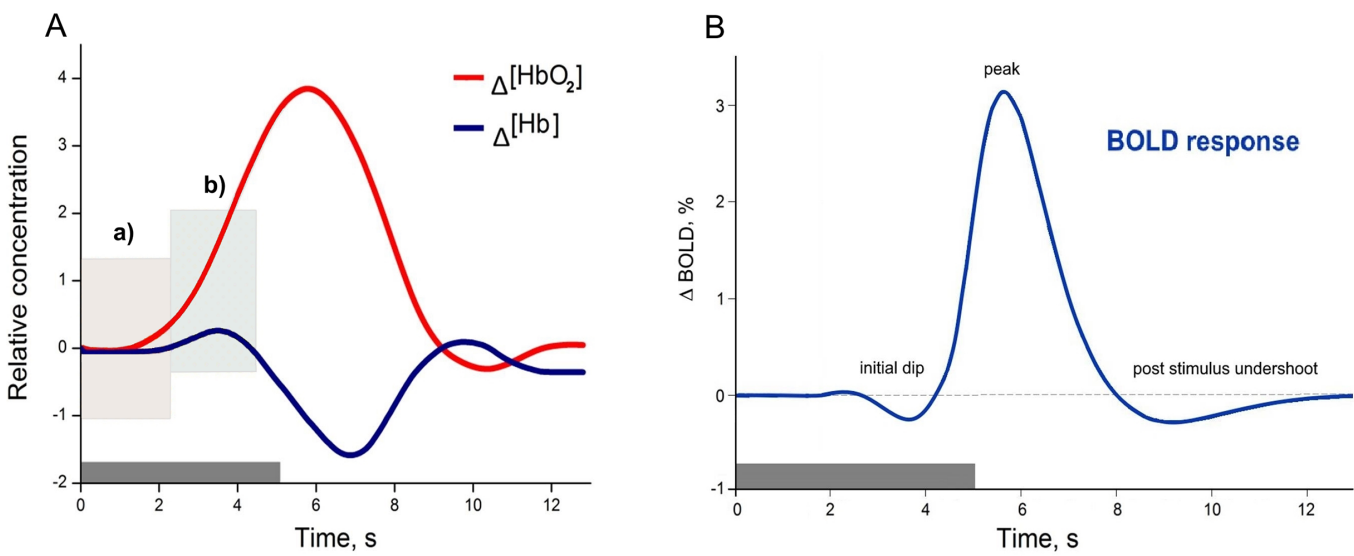

Figure 1 Examples of canonical hemodynamic response (A), and hemodynamic response function (B). A neural activity from 0 to $5 \mathrm{~s}$ (grey bar) causes neurometabolic and later neurovascular coupling, which can be seen as a delay of response (around $2 \mathrm{~s}$ ). Box in hemodynamic response (A) indicates (a) small inflow of $\Delta\left(\mathrm{HbO}_{2}\right)$, when the total blood volume is still relatively unchanged (due to increased cerebral blood flow), and later (b) $\Delta\left(\mathrm{HbO}_{2}\right)$ increases rapidly due to functional hyperemia caused by vasodilatation. The small increase of $\Delta(\mathrm{Hb})$ occurs due to insufficient washout when the cellular oxygen demand exceeds current supply in a tissue. The canonical example of a hemodynamic response is based on measuring the composition of cerebral blood volume via chromophore concentration changes (oxy-Hb and deoxy-Hb). fNIRS studies can directly measure both oxy-Hb and deoxy-Hb), (Venclove, Daktariunas \& Ruksenas, 2015). In contrast, the canonical hemodynamic response function (HRF) from the Blood Oxygenation Level Dependent (BOLD) method represents the magnetic field change in response to the $\Delta(\mathrm{Hb})$ curve (B) and is relative to the baseline.

Full-size

applicable. Thus only quite recently with technical progress fNIRS became an equivalent method for investigating human cognitive brain functions (Boas et al., 2014). Despite the common underlying phenomenon (Hillman, 2014; Huneau, Benali \& Chabriat, 2015; Iadecola, 2017; Phillips et al., 2016) both methods have their own strengths and limitations associated with biophysical and physiological signal origins (Kim \& Ogawa, 2012;

Scarapicchia et al., 2017). A comparison of how the same physiological process of NVC originates as a HR measured using fNIRS (A) and HR function measured using fMRI (B) can be found in Fig. 1. The example of HR (A), is based on measuring the composition of the total cerebral blood volume in the particular brain area. These measures can be directly done in vivo, and both oxygenated haemoglobin $\Delta\left(\mathrm{HbO}_{2}\right)$ and deoxygenated haemoglobin $\Delta(\mathrm{Hb})$ concentration changes are observed simultaneously. In contrast, the BOLD signal in fMRI is based on the paramagnetic deoxyhemoglobin decrease in T2* contrast, relative to the situation with diamagnetic oxyhemoglobin (Bandettini \& Wong, 1995; Chavhan et al., 2009). Thus, the $\Delta(\mathrm{Hb})$ curve in Fig. $1 \mathrm{~A}$ could be seen as a BOLD signal representation, even though it is not straightforward due to other physiological contributors captured in the BOLD response (such as regional cerebral blood flow and volume), (Arthurs \& Boniface, 2002; Kim \& Ogawa, 2012).

With technological progress, the number of combined functional fMRI-fNIRS studies has begun to increase slowly. According to the PubMed database search for the terms 'fMRI' and 'fNIRS' in the title/abstract field and restricted to the results of the articles and review papers published between January 1, 1977, and June 6, 2018, a total of 
752 documents with human participants were identified. After additional restriction to search for these terms only in the title field, a total of 11 documents remained (Anwar et al., 2013; Cui et al., 2011; Frederick, Nickerson \& Tong, 2012; Gagnon et al., 2012;

Maggioni et al., 2013; Okamoto et al., 2004; Sasai et al., 2012; Sato et al., 2013; Strangman et al., 2002; Tong \& Frederick, 2010; Toyoda et al., 2008). A few of them were comparisons, which have been conducted for a variety of cognitive tasks to illustrate similarities and differences between fNIRS and fMRI capabilities (Cui et al., 2011; Steinbrink et al., 2006). Overall, the described PubMed search serves as an illustrative example for two points: (a) number of multimodal, cross-validation, or comparison studies within even closely related techniques such as fMRI, and fNIRS is still insufficient; and (b) that integration of more distinct branches of neuroscience might be even more daring, but as will be discussed in the further section, might also add compelling value to the field of functional human brain research.

\section{Hemodynamic response and classical approach in neuroimaging}

The fundamentals of coupling between brain electrical activity, metabolism, and the observed HR are incredibly complex. Classical functional neuroimaging approaches for simplicity assume that the vascular response induced by neural activity is a nearly linear function of blood volume increase. This approach is convenient to model and reconstruct the possible neural activity from the HR but is not entirely accurate. Nonlinearities are believed to arise from both nonlinearities in the vascular response and neuronal activity, as several studies have demonstrated (Birn et al., 2013; Birn \& Bandettini, 2005; Hillman, 2014; Wager et al., 2005). Many more studies may be discussed depending on the reader's point of view, but the two following studies were chosen subjectively to provide a short illustrative introduction to this topic for non-experts. Wager et al. (2005) an empirical study with fMRI, where they attempt to characterise nonlinear effects in visual and motor cortex in 12 human participants, presents finding that these nonlinear effects are relatively consistent throughout the tested brain areas. Additionally, a more recent and exciting multimodal study by Fabiani et al. (2014) published in 2014 is recommended. In this multimodal study, 19 young and 44 older healthy adults were examined to address physical fitness and age effects on NVC in the primary visual cortex and show the quadratic relationship between neural activity and blood flow. The overall results indicate that nonlinearity in NVC has more than one aspect to be considered, and the classical neuroimaging approach is not sufficient to explain it.

What is the classical explanation of the origin of the HR? Over a decade, the understanding of NVC initiation and its overall role for HR formation dramatically changed (Attwell et al., 2010). From a CMN perspective, for a long time, researchers favoured the idea that blood flow is locally controlled by a passive negative-feedback system, where neural activity leads to a local substrate demand. It was thought that the metabolic signal inducing $\mathrm{HR}$ could be a lack of $\mathrm{O}_{2}$ or glucose, or the local rise of $\mathrm{CO}_{2}$, ADP or lactate. However, this negative-feedback hypothesis failed to adequately explain the experimental findings of NVC in animal models (Attwell et al., 2010; Walsh, 2016). Manipulations of $\mathrm{O}_{2}$ and glucose 
did not regulate blood flow as expected, and $\mathrm{CO}_{2}$, $\mathrm{ADP}$ together with lactate showed only partial effects (Attwell et al., 2010). More novel, feed-forwards neurotransmitter-mediated mechanisms suggest active control of the vascular energy supply in the brain (Attwell et al., 2010). In this process, the neurones either signal or activate astrocytes to release vasoactive messengers onto vessels. According to this hypothesis, astrocytes are anatomical intermediaries between neurones and blood vessels (Attwell et al., 2010; Petzold \& Murthy, 2011). However, is it all about astrocytes or other glial cells involved as well? Recent studies have begun to challenge the astrocytes role as the main drivers of NVC due to inconsistencies between spatiotemporal properties of vasodilatation, and the structure-functional properties and distribution of astrocytes in the cortical volume (Iadecola, 2017; McCaslin et al., 2011). In the following sub-section, the three examples of recent in vivo experiments will be discussed, to better illustrate the importance of non-neuronal HR origin.

\section{What induces a hemodynamic response and why does it matter?}

From a historical perspective 100 and 50 years ago the neuroglial was thought to be only a connective material in the brain and was given an entirely passive supportive role (Kettenmann \& Verkhratsky, 2008). Since then, a substantial amount of research has been published supporting the idea that the previous 'neuron-centric' perspective of neuroscience is not accurate. Today, it is evident that glial cells are integral to the development and maintenance of the healthy central nervous system and play a vital role in the pathogenesis of many brain disorders (Liddelow \& Barres, 2017).

The particular scientific focus was first given on astrocytes, as they are the most abundant population of glia in the mammalian brain (Azevedo et al., 2009; Liddelow \& Barres, 2017; Von Bartheld, Bahney \& Herculano-Houzel, 2016). It was proved that astrocytes are not only responsible for physical brain structuring but also are (a) critical homeostatic cellular elements that are capable of gluconeogenesis, provide neurones with lactate, and control over glucose levels (Bélanger, Allaman \& Magistretti, 2011; Brooks, 2009; Magistretti, 2006); (b) form a tripartite synapse with neurones and modulate synaptic activity via ion and neurotransmitters concentrations in the extracellular space (Allen \& Eroglu, 2017; Haydon \& Carmignoto, 2006; Krencik, Van Asperen \& Ullian, 2017; Perea \& Araque, 2005; Perea, Navarrete \& Araque, 2009); (c) they are responsible for some immune activity, promote neuronal survival and enable re-myelination within the brain (Ayaz et al., 2008; Liddelow \& Barres, 2017; Von Bernhardi, 2016); and (d) act as direct and indirect modulators of cerebrovascular tone (Ayata \& Lauritzen, 2015; Bazargani \& Attwell, 2016; Filosa et al., 2016; Gratton, Chiarelli \& Fabiani, 2017; Iadecola, 2017; Mishra, 2017). Moreover, they form an equivalent to neurones astroglial network (Attwell et al., 2010; Giaume et al., 2010; Scemes \& Spray, 2003).

After all, one may ask how these new cellular findings translate into functional neuroimaging. For example, the study of in vivo animal models (cat and rat) for a single-vessel hemodynamic demonstrated that pial surface arteries in the cat's visual cortex (as well as neurones) show orientation responsiveness (in contrast to rats, where orientation maps are not shown in general), meaning that propagation of vascular 
dilation between neighbouring columns in the brain needs to be accounted for when decoding hemodynamic signals (O'Herron et al., 2016).

Another in vivo study of animal models (rat and mice) show that when the sensory input increases, blood flow capillaries dilate before arterioles and are estimated to produce $84 \%$ of the blood flow increase (Hall et al., 2014). Previously, it was thought that capillaries usually do not significantly contribute. Moreover, the study identifies pericytes as significant regulators of cerebral blood flow as they are the first vascular elements to dilate during neuronal activity, and, in turn, initiate hyperaemia. It also unexpectedly showed that vasodilators released from active neurones, interneurones and astrocytes (Hamel, 2006; Miller \& Halpern, 2014) are not the only essential players in functional imaging. In fact, the role of pericytes in CNS is as diverse as it was previously described with astrocytes: pericytes integrate, coordinate and process signals from their neighbouring cells to generate diverse functional responses that are critical for CNS functions in health and disease, including (a) regulation of the blood-brain barrier (BBB) permeability; (b) angiogenesis; (c) clearance of toxic metabolites; (d) neuroinflammation and stem cell activity; and finally (e) initiating capillary HRs (Hall et al., 2014; Iadecola, 2017; Kisler et al., 2017; Sweeney, Ayyadurai \& Zlokovic, 2016).

Also, another non-neuronal cell type crucial for inducing HR was recently identifiedvascular endothelium. Several kinds of research demonstrated that vascular endothelium could propagate upstream dilations of cerebral vessels (Andresen, Shafi \& Bryan, 2006; Chen et al., 2011; Hannah et al., 2011; Iadecola, 2017; Rosenblum, 1986). The in vivo study by Chen et al. (2014), demonstrated that spatially selective endothelial disruption with light-dye treatment in rats somatosensory cortex significantly attenuated the HR by blocking the retrograde dilation. The early stage and the peak of hyperaemia were affected the most, meaning that neurones, astrocytes, pericytes and endothelial cells are all involved in forming HR detected by functional neuroimaging.

There are many other scientific sources regarding cellular, molecular biology and NVC that could be discussed. However, even with the given three in vivo examples, it is evident that the main drivers of NVC, and temporal properties of HR associated with it, depends on the spatial location along the cerebral vasculature (Iadecola, 2017). These new findings allow re-evaluating, how spatiotemporal specificity may be improved alongside the technological progress of fNIRS and fMRI. Because ultimately, the non-invasive use of $\mathrm{HR}$ is one of the most powerful tools at our disposal to explore human cognition in health and disease.

\section{ACCOUNTING FOR THE COMPLEXITY OF CEREBROVASCULAR REGULATION}

The HR describes the empirical observation of a physiological NVC event. It may be detected as an amplitude changes in light absorption (fNIRS) or as a magnetic signal variation (fMRI). In other words, a $\mathrm{HR}$ is a spatiotemporal picture of underlying NVC and cerebrovascular regulation at large. To better understand this, some structural and functional properties of cerebrovasculature must be explained. 


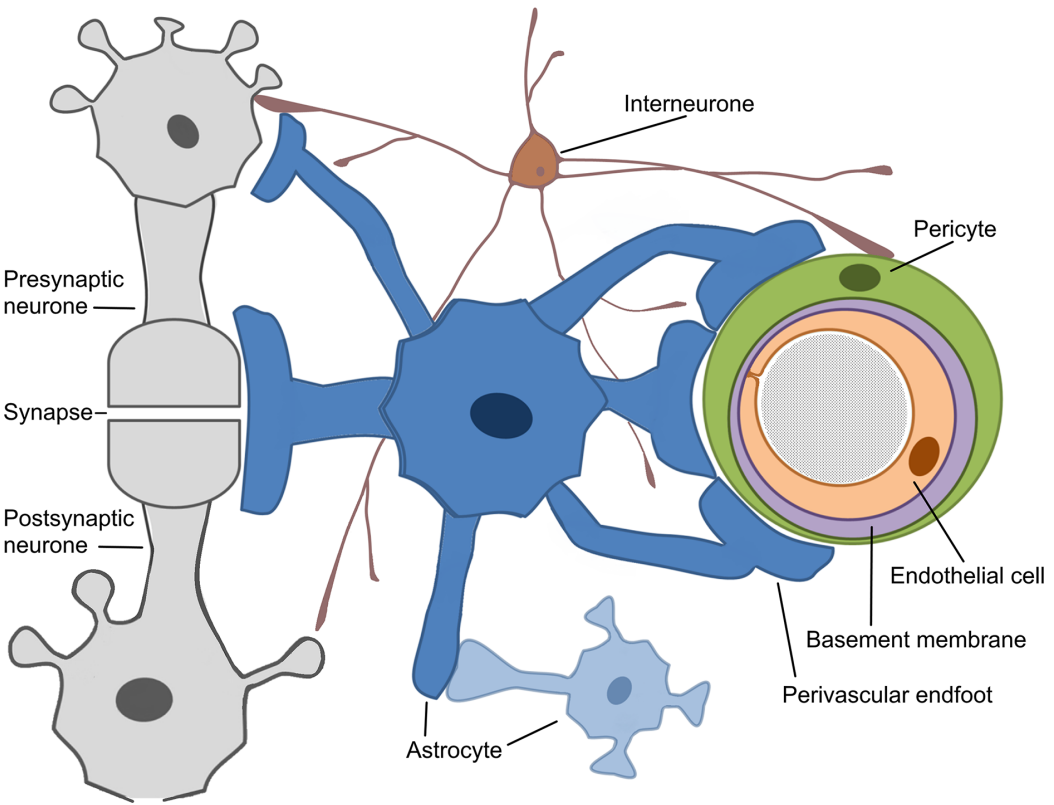

Figure 2 Schematic representation of the Neurovascular Unit (NVU).

Full-size $\underset{0}{0}$ DOI: 10.7717 /peerj.6621/fig-2

\section{Neurovascular unit}

The concept of the neurovascular unit (NVU) emerged around 2001 (Iadecola, 2017). The whole mechanism of cerebrovascular regulation can generally be decomposed into several stages (Hamel, 2006; Phillips et al., 2016; Walsh, 2016). The most explored cerebral microcirculation is provided by the structural and functional derivative called NVU, (Attwell et al., 2010; Leybaert, 2005). The neurovascular unit represents the interface between the vascular and neural compartments in the brain and is composed of vascular, glial and neuronal cells such as neurones, astrocytes, endothelial cells and pericytes (Hawkins \& Davis, 2005; Sweeney, Ayyadurai \& Zlokovic, 2016; Sweeney, Walker-Samuel \& Shipley, 2018), (Fig. 2). The NVU is an essential structure for several main processes: formation of neurometabolic coupling (NMC), NVC and formation of the BBB, (Leybaert, 2005; Leybaert et al., 2007; Petzold \& Murthy, 2011). NVU may vary in structure and function depending on its location in the brain (Iadecola, 2017; Kowianski et al., 2013; Petzold \& Murthy, 2011), thus emphasising the complexity of numerous processes that are involved in maintaining adequate blood flow in the healthy human brain. Neuronal activity in the brain causes two cerebrovascular regulation processes: NMC, which undergoes a substrate exchange between a neurone and an astrocyte and later initiates (but not necessarily) NVC. NVU also supports the BBB coupling but is believed to be unbundled from the already mentioned $\mathrm{NBC}$ and $\mathrm{NVC}$, as it regulates the integrity and functions of the BBB (Leybaert, 2005; Sweeney, Ayyadurai \& Zlokovic, 2016). New pieces of evidence suggest that another critical component of the NVU are the interneurones that transduce signals from perivascular nerves (Hamel, 2006; Walsh, 2016), (Fig. 2). The crucial role of the perivascular nerves is to regulate the cerebrovascular tone influencing the overall brain perfusion. NVC is then determined by the chemical 
signals released from the activated perivascular nerves and astrocytes, and together alter the vascular tone to adjust local perfusion in accordance with the brain activity (Hamel, 2006; Walsh, 2016).

\section{Signal transduction path in the neurovascular unit}

With progress in molecular and cellular biology, a conceptual shift in our understanding of cerebral blood flow occurred where astrocytes, previously believed to be passive supporting cells, had been shown to actively participate in many other physiological processes, as well as creating equivalent to neural to the astroglial network, and directly modulating neural activity (Giaume et al., 2010; Kowiański et al., 2013; Scemes \& Spray, 2003). For a while, the idea that elevations of calcium concentration in the astrocytes may release transmitters that regulate neuronal and vascular functions was controversial (Barres, 2008; Bezzi et al., 2004; Fiacco, Agulhon \& McCarthy, 2009). This changed when numerous contradictions were reported between different studies and had been resolved (Bazargani \& Attwell, 2016). Shortly after the discovery that glutamate triggers an increase of intracellular calcium concentration $\left(\left[\mathrm{Ca}^{2+}\right]_{i}\right)$, it was suggested that there might be a mechanism by which calcium signalling propagates towards astrocyte's endfeet and stimulates the release of vasoactive messengers (Attwell et al., 2010). Vasoactive messengers can cause vasodilatation (most of the neurotransmitters; nitric oxide; prostaglandins; epoxyeicosatrienoic acids; lactate; adenosine etc.) or vasoconstriction (norepinephrine; 20-Hydroxyeicosatetraenoic acid etc.) of arterioles (Giaume et al., 2010; Kowianski et al., 2013; Leybaert, 2005; Petzold \& Murthy, 2011; Scemes \& Spray, 2003). Current understanding suggests that astrocytes, as well as neurones, should be divided into three spatial compartments, such as processes, soma and endfeet (Bazargani \& Attwell, 2016). In this way, the release of specific vasoactive messengers in the endfeet is explained by an overall summation of $\left(\left[\mathrm{Ca}^{2+}\right]_{i}\right)$ in the soma and processes. The response may differ in terms of frequency, kinetics, spatial spreads and interaction with other cellular messengers (Bazargani \& Attwell, 2016). As was previously discussed, astrocytes are not the only cells that are involved in $\mathrm{HR}$, but they continue to be considered the main drivers of NVC.

Because of this, the article proposes the conceptual biophysical scheme of the biological signal transduction path in the neurovascular unit (Fig. 3), as a brief adaptation of the classical approach of NVU. Figure 3 summarises how metabolic and physiological events (NMC and NVC accordingly) via calcium concentration elevation cause the HR. This, in turn, can be observed with functional neuroimaging. Note that the conceptual biophysical scheme inevitably assumes neural activity-derived NVC. Nonetheless, recent findings show that astroglial metabolic networks may sustain or suppress neuronal activity (Giaume et al., 2010). For simplicity, this article does not account for it or discuss it thoroughly, as more studies have to be done to generalise new findings (Giaume et al., 2010; Walsh, 2016). However, the idea should be kept in mind for the critical evaluation of current functional neuroimaging methods, and the proposed scheme should be used as a tool for a brief explanation of how signal transduction for cerebrovascular regulation occurs in NVU. Previously mentioned evidence of spatiotemporal specificity of NVU are 


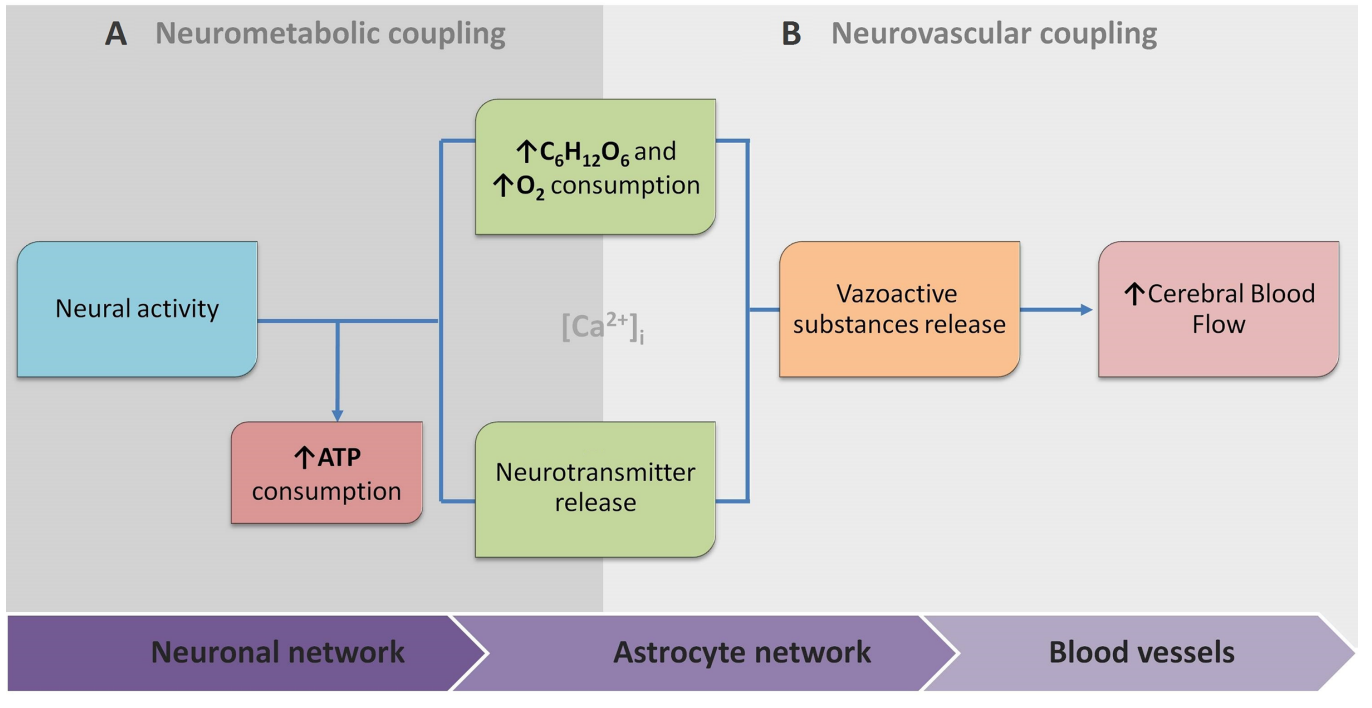

Figure 3 The conceptual biophysical scheme of biological signal transduction path in the Neurovascular Unit (NVU). (A) Neurometabolic coupling (NMC); (B) Neurovascular coupling (NVC). Both neurones (neurotransmitter release) and astrocytes (glucose and oxygen consumption) respond to increased extracellular glutamate, and intracellular calcium to transmit direct and indirect vasoactive signals for the appropriate blood delivery and distribution in the electrically active brain area. Full-size

not included to avoid unnecessary complexity as information about additional players in coupling is still under investigation.

The same complexity of cerebrovascular regulation raised the developmental of numerous approaches, ranging from purely statistical signal processing to biophysical modelling at various levels of detail. Regional hemodynamic changes measured by fNIRS and fMRI are modelled separately because of different aspects of HR that are captured, and sources of noise that are involved. Regarding fNIRS, the extended version of an existing computational model of cerebral physiology, 'BrainSignals' should be considered as the most prominent (Caldwell et al., 2016). It incorporates components of

(a) hemodynamic; (b) mitochondrial brain metabolism (c) brain oxygen consumption; and (d) scalp hemodynamic. This model also joins hemoglobin-based and the cytochrome c oxidase redox state based measurements (which are out of scope of this article but are promising branch of optical brain measurements). Moreover, the authors compare their empirical model with real measurements, which give promising results in detecting non-linear confounding effects, which are also extensively highlighted by other authors (Tachtsidis \& Scholkmann, 2016).

Meanwhile, most models of BOLD response are based solely on cerebral blood flow, cerebral blood volume, and the local metabolic rate of oxygen consumption (Kim \& Ogawa, 2012; Toga, 2015). These models depict the transient hemodynamic, and oxygenation changes in the activated cerebral areas also mimic some of the physiological mechanisms of functional hyperemia and are extensively discussed by Huneau, Benali \& Chabriat (2015). Authors shortly state, that despite the accumulation of new findings, NVC has surprisingly been forsaken in modelling functional neuroimaging, especially in 
humans. On the other hand, the field of mathematical modelling of BOLD reached some significant consensus across variables that should be involved in a generative hemodynamic model (using dynamic causal modelling approach), (Havlicek et al., 2015). It involves several different models, such as (a) neuronal; (b) NVC; (c) hemodynamic; and (d) BOLD in a joint model. The approach reflects experimental observations of underlying physiological processes and corresponds well with multimodal experimental datasets (Havlicek et al., 2017).

Meanwhile, technological improvement of neuroimaging techniques allows creating new and more specific models for investigating NVC. For example, a novel in vivo study combining imaging of cortical microvascular and mural cell architecture together with mathematical modelling of blood flow and oxygen transport provided new insights on seemingly paradoxical observations in the literature around reduced blood velocity in response to arteriolar constrictions, and found that it might be caused by propagation of constrictions to upstream penetrating arterioles (Sweeney, Walker-Samuel \& Shipley, 2018). A similar investigation of cerebral blood flow (CBF) regulation would be inaccessible in a conventional experimental context. In this study, results were achieved by using in vivo collected information for in silico experimentation.

\section{WHY FOCUSED INTERDISCIPLINARY INTEGRATION SHOULD MATTER}

How does one determine whether HR under neurological or psychiatric conditions reflects underlying neural activity rather than altered NVC? Does it mean that in many cases additional validation studies linking neuronal activity with NVC might be needed to rely on cognitive inferences derived from functional neuroimaging entirely? A growing body of evidence from animal studies suggests it (Lindauer et al., 2010; O'Herron et al., 2016). Other questions like (i) how new findings of non-neural cell interactions change the interpretation of neural activity derived from previous functional neuroimaging, and (ii) how to distinguish between neural-activity-derived and only glia-activity-derived hemodynamic events, remain open.

Neuroscience is a multidisciplinary branch of biology, and its scope has broadened over time to include a lot of new and different approaches in many aspects of the nervous system. As a result, neuroscience exploded in a number of interdisciplinary fields such as neurophysiology, cognitive and behavioural neuroscience, computational neuroscience and translational neuroscience research. Somehow, common researchers' knowledge assumes that in such a broad community of neuroscientists and clinicians, there must be enough professionals dedicating their time and effort to some issues and that necessary integration will occur naturally at the particular threshold. However, some main conceptual challenges may remain daunting due to an unfocused, one-side-driven interdisciplinary integration. Illustrating it with terms of symbiosis: when relationships and interactions between different branches of neuroscience are based more on commensalism (when part A benefits from part B, but B remain unaffected), rather than mutualism (when part A benefits from part B, and vice versa). Of course, interdisciplinary integrations are way more complicated, but some relevant patterns may be observed. 


\section{TIMELINE OF MAJOR EVENTS}

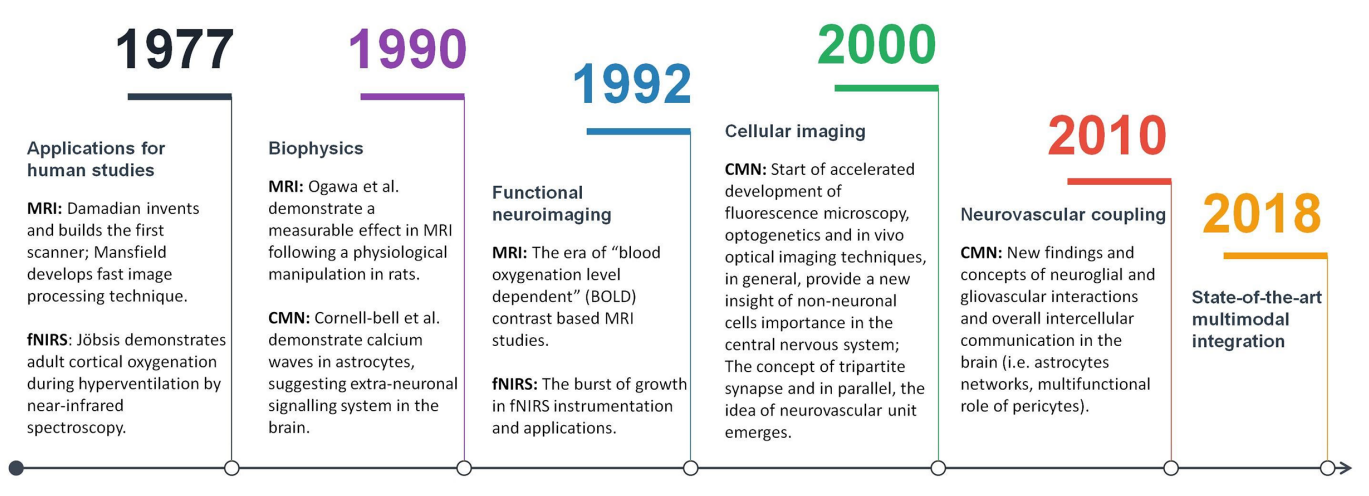

Figure 4 A timeline of magnetic resonance imaging (MRI), functional near-infrared spectroscopy (fNIRS), and cellular and molecular neuroscience (CMN) milestones.

Full-size

For example, a few historical moments of fMRI, fNIRS and CMN are given in a single timeline (Fig. 4). As can be seen from Fig. 4, some significant milestones, such as a burst of functional neuroimaging studies using fMRI and fNIRS were achieved simultaneously around 1992. While others, conceptually very important, such as the concepts of the tripartite synapse and neurovascular unit, emerged only around 2000. There is no surprise in the different timing between different neuroscience branch achievements in general. However, even after more than a decade following seminal research in 2000, the NVC is still surprisingly overlooked in functional neuroimaging modelling, especially in humans. This means that current functional neuroimaging inference guidelines are poorly addressing even already known findings of underlying physiological mechanisms of NVC. This renders further interpretations of the HR based functional neuroimaging results ambiguous and entirely reliable only with support from anatomical and electrophysiological studies. As is often the case, the primary concern is not the validity of previous and current studies using functional neuroimaging, but advancements and innovation in the existing paradigm. Ultimately, the non-invasive use of $\mathrm{HR}$ is one of the most powerful tools at our disposal to explore human cognition in health and disease, and thus the focused interdisciplinary attention on its accuracy should matter to anyone working or with interests in the field of human brain research.

\section{CONCLUSIONS AND FUTURE PERSPECTIVES}

The significant part of scientific and clinical research production is based on complex mathematical manipulations of neuroimaging data derived from NVC. To improve the overall quality of this production, a complete interpretation of HR should become a number one concern in the field, as it is the primary information source of underlying human brain neurophysiology. However, the amount of available information is growing exponentially, and due to this information overload, researchers' attention span may be 
naturally decreased, thus making it a definite obstacle. On the other hand, as was illustrated with the PubMed database search, even within closely related techniques such as fMRI, and fNIRS, there is evident lack of close integration. Moreover, significant results from even more distinct branches of neuroscience such as cellular biology and in vivo brain physiology are instead suggested for consideration than provided for implementing in existing CM of human brain function.

One way to overcome it is to stress the concern and make it easy to perceive for broader scientific communities. In accordance, this article fills the gap of a critical view on HR translation into scientific findings and expresses the need for more similarly focused interdisciplinary reviews, as numerous aspects cannot be thoroughly generalised at once. Also, it addresses the need to integrate neurophysiology and computational neuroscience fields to stimulate innovations in neuroimaging by improving an accurate translation of physiological brain signals. At this point, another possible suggestion would be to implement existing machine learning (ML) algorithms for data mining. It would allow meticulous comparison of existing data in CMN studies of NVC, and functional neuroimaging.

In contrast to existing approaches (which use sophisticated algorithms to perform large-scale medical data analysis to search for patterns and predictions in certain brain diseases), attention could be focused on the problem of translating complex physiological phenomena (NVC) to functional neuroimaging (brain maps of HR). This analytical approach in biomedicine is successfully implemented elsewhere (Cao et al., 2018; Ching et al., 2018). In fact, according to latest report of artificial intelligence (AI) use (Shoham et al., 2018), the significant portion of AI-technology-based papers in the USA and Europe tend to be focused on the humanities, and medical and health sciences. Unfortunately, no such attempt was found in the current literature concerning the HR. The existing approaches, from previous (Banaji et al., 2008; Buxton, Wong \& Frank, 1998; Caldwell et al., 2016; Friston et al., 2000; Huneau, Benali \& Chabriat, 2015; Sotero \& Trujillo-Barreto, 2007, 2008), to more recent (Havlicek et al., 2017; Sweeney, Walker-Samuel \& Shipley, 2018) models are exploratory, meaning that they try to determine, whether what is being observed might be explained by a currently existing theory. Further, an analytical approach with ML algorithms could be used for patterning and prediction in a conceptually different manner. Despite the notable advantages, it is important to note that applying it would be inevitably challenging; mostly because of data properties, as several different approaches might be needed at once (Cao et al., 2018; Ching et al., 2018). Meanwhile, the author suggests a few general points to discuss on how necessary integration could be initiated:

(a) Systematic reviews and meta-analyses of previous research studies could be performed selectively on different aspects of NVC and HR (i.e. modality used to investigate, species of a subject, spatial location of interest in the brain volume, goals of research and employed pharmacological agents). The literature search could be expanded with AI algorithms dedicated for the search of relevant scientific content with extensive vocabulary from different neuroscience fields to avoid losing information, when 
publications conceptually are about the same physiological phenomenon, but due to historical context, or other reasons, is described differently (like terms HR and HR function). This would ensure unbiased (by the investigators' prior knowledge) collection of relevant publications. As an existing equivalent could be considered AI2 system by the Allen Institute, called 'Semantic Scholar', dedicated to finding peerreviewed research from only trusted, verified sources (https://www.semanticscholar. org). Another example-Elsevier Fingerprint Engine,-the same systems that were applied to explore previously mentioned AI tendency to focus on healthcare. It used a primary set of about 800 keywords relevant to AI (Shoham et al., 2018). Other engines that are not mentioned in this publication may also be used directly or as a prototype. After the initial search on particular aspects of NCV and HR (mostly to make easier the quality check before further analysis), the data could be combined and sliced by any relevant dimension. With a sufficient quantity of information, several different data mining approaches could be possibly applied.

(b) The initial collection for systematic reviews and meta-analyses could be reused to build a database (or a platform with some user interface) and a unified template for a new data entry could be created, as a suggestion what metadata file that could be associated with a new publication. It would make it easier to import new data and this in turn would increase publication's impact and visibility. Some concepts of similar systems could also be borrowed and implemented from already existing projects such as the Human Brain Project (https://www.humanbrainproject.eu), or maybe even branch out as a separate compartment of an already existing platform.

(c) This newly created database could also serve as an information source for any level of computational insight: CM, deep learning, ML, or AI. The database could provide some guidelines for the researchers when searching, or preparing training data for their in silico experimentation. The purpose of mentioned algorithms may vary from an automated classification of inputs from living cell microarrays (Jonczyk et al., 2016) to sophisticated machine learning algorithms searching for discrepancies across multimodal studies as in previously given examples.

To sum up, most of the perspective tools are already available and needs only to be implemented with a particular problem. A broader and multi-disciplinary appreciation of NVC could further boost basic and clinical neuroscience. Thus, a reason why it is still not in the frontlines of functional neuroimaging remains debatable. On another hand, this gap in neuroscience requires state-of-the-art scientific research. Because of this, the author invites scientific researches to respond in comments or with a follow-up publication and propose tools or strategies that could be implemented towards accurate translation of the HR.

\section{ACKNOWLEDGEMENTS}

The author would like to thank colleagues A. Daktariunas and O. Ruksenas for contributing with helpful ideas. The author wishes to thank the editor and the reviewers for their contribution. 


\section{ADDITIONAL INFORMATION AND DECLARATIONS}

\section{Funding}

The author received no funding for this work.

\section{Competing Interests}

The author declare having no competing interests.

\section{Author Contributions}

- Sigita Cinciute conceived and designed the experiments, performed the experiments, analysed the data, contributed reagents/materials/analysis tools, prepared figures and/or tables, authored or reviewed drafts of the paper, approved the final draft.

\section{Data Availability}

The following information was supplied regarding data availability:

The research in this article did not generate any data or code (review article).

\section{REFERENCES}

Allen NJ, Eroglu C. 2017. Cell biology of astrocyte-synapse interactions. Neuron 96(3):697-708 DOI 10.1016/j.neuron.2017.09.056.

Andresen J, Shafi NI, Bryan RM. 2006. Endothelial influences on cerebrovascular tone. Journal of Applied Physiology 100(1):318-327 DOI 10.1152/japplphysiol.00937.2005.

Anwar AR, Muthalib M, Perrey S, Galka A, Granert O, Wolff S, Deuschl G, Raethjen J, Heute U, Muthuraman M. 2013. Comparison of causality analysis on simultaneously measured fMRI and NIRS signals during motor tasks. In: Proceedings of the Annual International Conference of the IEEE Engineering in Medicine and Biology Society. Piscataway: IEEE, 2628-2631.

Arthurs OJ, Boniface S. 2002. How well do we understand the neural origins of the fMRI BOLD signal? Trends in Neurosciences 25(1):27-31 DOI 10.1016/S0166-2236(00)01995-0.

Attwell D, Buchan AMA, Charpak S, Lauritzen M, MacVicar BBA, Newman EEA. 2010. Glial and neuronal control of brain blood flow. Nature 468(7321):232-243 DOI 10.1038/nature09613.

Ayata C, Lauritzen M. 2015. Spreading depression, spreading depolarizations, and the cerebral vasculature. Physiological Reviews 95(3):953-993 DOI 10.1152/physrev.00027.2014.

Ayaz H, Allen SL, Platek SM, Onaral B. 2008. Maze Suite 1.0: a complete set of tools to prepare, present, and analyze navigational and spatial cognitive neuroscience experiments. Behavior Research Methods 40(1):353-359 DOI 10.3758/BRM.40.1.353.

Azevedo FAC, Carvalho LRB, Grinberg LT, Farfel JM, Ferretti REL, Leite REP, Filho WJ, Lent R, Herculano-Houzel S. 2009. Equal numbers of neuronal and nonneuronal cells make the human brain an isometrically scaled-up primate brain. Journal of Comparative Neurology 513(5):532-541 DOI 10.1002/cne.21974.

Banaji M, Mallet A, Elwell CE, Nicholls P, Cooper CE. 2008. A model of brain circulation and metabolism: NIRS signal changes during physiological challenges. PLOS Computational Biology 4(11):e1000212 DOI 10.1371/journal.pcbi.1000212.

Bandettini PA, Wong EC. 1995. Effects of biophysical and physiologic parameters on brain activation-induced $\mathrm{R} 2^{*}$ and $\mathrm{R} 2$ changes: simulations using a deterministic diffusion model. International Journal of Imaging Systems and Technology 6(2-3):133-152 DOI 10.1002/ima.1850060203. 
Barres BA. 2008. The mystery and magic of glia: a perspective on their roles in health and disease. Neuron 60(3):430-440 DOI 10.1016/j.neuron.2008.10.013.

Bazargani N, Attwell D. 2016. Astrocyte calcium signaling: the third wave. Nature Neuroscience 19(2):182-189 DOI 10.1038/nn.4201.

Bélanger M, Allaman I, Magistretti PJ. 2011. Brain energy metabolism: focus on astrocyte-neuron metabolic cooperation. Cell Metabolism 14(6):724-738 DOI 10.1016/j.cmet.2011.08.016.

Bezzi P, Gundersen V, Galbete JL, Seifert G, Steinhäuser C, Pilati E, Volterra A. 2004. Astrocytes contain a vesicular compartment that is competent for regulated exocytosis of glutamate. Nature Neuroscience 7(6):613-620 DOI 10.1038/nn1246.

Birn RM, Bandettini PA. 2005. The effect of stimulus duty cycle and "off" duration on BOLD response linearity. NeuroImage 27(1):70-82 DOI 10.1016/j.neuroimage.2005.03.040.

Birn RM, Molloy EK, Patriat R, Parker T, Meier TB, Kirk GR, Nair VA, Elizabeth Meyerand M, Prabhakaran V. 2013. The effect of scan length on the reliability of resting-state fMRI connectivity estimates. NeuroImage 83:550-558 DOI 10.1016/j.neuroimage.2013.05.099.

Boas DA, Elwell CE, Ferrari M, Taga G. 2014. Twenty years of functional near-infrared spectroscopy: introduction for the special issue. NeuroImage 85:1-5 DOI 10.1016/j.neuroimage.2013.11.033.

Brooks GA. 2009. Cell-cell and intracellular lactate shuttles. Journal of Physiology 587(23):5591-5600 DOI 10.1113/jphysiol.2009.178350.

Buxton RB, Wong EC, Frank LR. 1998. Dynamics of blood flow and oxygenation changes during brain activation: the balloon model. Magnetic Resonance in Medicine 39(6):855-864 DOI 10.1002/mrm.1910390602.

Caldwell M, Scholkmann F, Wolf U, Wolf M, Elwell C, Tachtsidis I. 2016. Modelling confounding effects from extracerebral contamination and systemic factors on functional near-infrared spectroscopy. NeuroImage 143:91-105 DOI 10.1016/j.neuroimage.2016.08.058.

Cao C, Liu F, Tan H, Song D, Shu W, Li W, Zhou Y, Bo X, Xie Z. 2018. Deep learning and its applications in biomedicine. Genomics, Proteomics \& Bioinformatics 16(1):17-32 DOI 10.1016/j.gpb.2017.07.003.

Chavhan GB, Babyn PS, Thomas B, Shroff MM, Haacke EM. 2009. Principles, techniques, and applications of T2*-based MR imaging and its special applications. RadioGraphics 29(5):1433-1449 DOI 10.1148/rg.295095034.

Chen BR, Bouchard MB, McCaslin AFH, Burgess SA, Hillman EMC. 2011. High-speed vascular dynamics of the hemodynamic response. NeuroImage 54(2):1021-1030

DOI 10.1016/j.neuroimage.2010.09.036.

Chen BR, Kozberg MG, Bouchard MB, Shaik MA, Hillman EMC. 2014. A critical role for the vascular endothelium in functional neurovascular coupling in the brain. Journal of the American Heart Association 3(3):e000787 DOI 10.1161/JAHA.114.000787.

Ching T, Himmelstein DS, Beaulieu-Jones BK, Kalinin AA, Do BT, Way GP, Ferrero E, Agapow P-M, Zietz M, Hoffman MM, Xie W, Rosen GL, Lengerich BJ, Israeli J, Lanchantin J, Woloszynek S, Carpenter AE, Shrikumar A, Xu J, Cofer EM, Lavender CA, Turaga SC, Alexandari AM, Lu Z, Harris DJ, DeCaprio D, Qi Y, Kundaje A, Peng Y, Wiley LK, Segler MHS, Boca SM, Joshua Swamidass S, Huang A, Gitter A, Greene CS. 2018. Opportunities and obstacles for deep learning in biology and medicine. Journal of the Royal Society Interface 15(141):20170387 DOI 10.1098/rsif.2017.0387.

Cui X, Bray S, Bryant DM, Glover GH, Reiss AL. 2011. A quantitative comparison of NIRS and fMRI across multiple cognitive tasks. NeuroImage 54(4):2808-2821

DOI 10.1016/j.neuroimage.2010.10.069. 
Fabiani M, Gordon BA, Maclin EL, Pearson MA, Brumback-Peltz CR, Low KA, McAuley E, Sutton BP, Kramer AF, Gratton G. 2014. Neurovascular coupling in normal aging: a combined optical, ERP and fMRI study. NeuroImage 85(5):592-607

DOI 10.1016/j.neuroimage.2013.04.113.

Fiacco TA, Agulhon C, McCarthy KD. 2009. Sorting out astrocyte physiology from pharmacology. Annual Review of Pharmacology and Toxicology 49(1):151-174 DOI 10.1146/annurev.pharmtox.011008.145602.

Filosa JA, Morrison HW, Iddings JA, Du W, Kim KJ. 2016. Beyond neurovascular coupling, role of astrocytes in the regulation of vascular tone. Neuroscience 323(5):96-109 DOI 10.1016/j.neuroscience.2015.03.064.

Frederick B, Nickerson LD, Tong Y. 2012. Physiological denoising of BOLD fMRI data using regressor interpolation at progressive time delays (RIPTiDe) processing of concurrent fMRI and near-infrared spectroscopy (NIRS). NeuroImage 60(3):1913-1923

DOI 10.1016/j.neuroimage.2012.01.140.

Friston KJ, Mechelli A, Turner R, Price CJ. 2000. Nonlinear responses in fMRI: the balloon model, Volterra Kernels, and other Hemodynamics. NeuroImage 12(4):466-477 DOI 10.1006/nimg.2000.0630.

Gagnon L, Yücel MA, Dehaes M, Cooper RJ, Perdue KL, Selb J, Huppert TJ, Hoge RD, Boas DA. 2012. Quantification of the cortical contribution to the NIRS signal over the motor cortex using concurrent NIRS-fMRI measurements. NeuroImage 59(4):3933-3940 DOI 10.1016/j.neuroimage.2011.10.054.

Giaume C, Koulakoff A, Roux L, Holcman D, Rouach N. 2010. Astroglial networks: a step further in neuroglial and gliovascular interactions. Nature Reviews Neuroscience 11(2):87-99 DOI $10.1038 /$ nrn2757.

Glover GH. 2011. Overview of functional magnetic resonance imaging. Neurosurgery Clinics of North America 22(2):133-139 DOI 10.1016/j.nec.2010.11.001.

Gratton G, Chiarelli AM, Fabiani M. 2017. From brain to blood vessels and back: a noninvasive optical imaging approach. Neurophotonics 4(3):031208 DOI 10.1117/1.NPh.4.3.031208.

Hall CN, Reynell C, Gesslein B, Hamilton NB, Mishra A, Sutherland BA, O'Farrell FM, Buchan AM, Lauritzen M, Attwell D. 2014. Capillary pericytes regulate cerebral blood flow in health and disease. Nature 508(7494):55-60 DOI 10.1038/nature13165.

Hamel E. 2006. Perivascular nerves and the regulation of cerebrovascular tone. Journal of Applied Physiology 100(3):1059-1064 DOI 10.1152/japplphysiol.00954.2005.

Hannah RM, Dunn KM, Bonev AD, Nelson MT. 2011. Endothelial SKCa and IKCa Channels regulate brain parenchymal arteriolar diameter and cortical cerebral blood flow.

Journal of Cerebral Blood Flow \& Metabolism 31(5):1175-1186 DOI 10.1038/jcbfm.2010.214.

Hansen MM, Miron-Shatz T, Lau AYS, Paton C. 2014. Big data in science and healthcare: a review of recent literature and perspectives. Yearbook of Medical Informatics 23(1):21-26 DOI 10.15265/IY-2014-0004.

Havlicek M, Ivanov D, Roebroeck A, Uludağ K. 2017. Determining excitatory and inhibitory neuronal activity from multimodal fMRI data using a generative hemodynamic model. Frontiers in Neuroscience 11:1-20 DOI 10.3389/fnins.2017.00616.

Havlicek M, Roebroeck A, Friston K, Gardumi A, Ivanov D, Uludag K. 2015. Physiologically informed dynamic causal modeling of fMRI data. NeuroImage 122:355-372 DOI 10.1016/j.neuroimage.2015.07.078.

Hawkins B, Davis T. 2005. The blood-brain barrier/neurovascular unit in health and disease. Pharmacological Reviews 57(2):173-185 DOI 10.1124/pr.57.2.4. 
Haydon PGP, Carmignoto G. 2006. Astrocyte control of synaptic transmission and neurovascular coupling. Physiological Reviews 86(3):1009-1031 DOI 10.1152/physrev.00049.2005.

Herculano-Houzel S. 2011. Scaling of brain metabolism with a fixed energy budget per neuron: implications for neuronal activity, plasticity and evolution. PLOS ONE 6(3):e17514 DOI 10.1371/journal.pone.0017514.

Hillman EMCC. 2014. Coupling mechanism and significance of the BOLD signal: a status report. Annual Review of Neuroscience 37(1):161-181 DOI 10.1146/annurev-neuro-071013-014111.

Huneau C, Benali H, Chabriat H. 2015. Investigating human neurovascular coupling using functional neuroimaging: a critical review of dynamic models. Frontiers in Neuroscience 9:467 DOI 10.3389/fnins.2015.00467.

Iadecola C. 2017. The neurovascular unit coming of age: a journey through neurovascular coupling in health and disease. Neuron 96(1):17-42 DOI 10.1016/j.neuron.2017.07.030.

Jonczyk R, Kurth T, Lavrentieva A, Walter J-G, Scheper T, Stahl F. 2016. Living cell microarrays: an overview of concepts. Microarrays 5(2):11 DOI 10.3390/microarrays5020011.

Kettenmann H, Verkhratsky A. 2008. Neuroglia: the 150 years after. Trends in Neurosciences 31(12):653-659 DOI 10.1016/j.tins.2008.09.003.

Kim S-G, Ogawa S. 2012. Biophysical and physiological origins of blood oxygenation level-dependent fMRI signals. Journal of Cerebral Blood Flow \& Metabolism 32(7):1188-1206 DOI 10.1038/jcbfm.2012.23.

Kisler K, Nelson AR, Rege SV, Ramanathan A, Wang Y, Ahuja A, Lazic D, Tsai PS, Zhao Z, Zhou Y, Boas DA, Sakadžić S, Zlokovic BV. 2017. Pericyte degeneration leads to neurovascular uncoupling and limits oxygen supply to brain. Nature Neuroscience 20(3):406-416 DOI 10.1038/nn.4489.

Kowiański P, Lietzau G, Steliga A, Waśkow M, Moryś J. 2013. The astrocytic contribution to neurovascular coupling-still more questions than answers? Neuroscience Research 75(3):171-183 DOI 10.1016/j.neures.2013.01.014.

Krencik R, Van Asperen JV, Ullian EM. 2017. Human astrocytes are distinct contributors to the complexity of synaptic function. Brain Research Bulletin 129:66-73 DOI 10.1016/j.brainresbull.2016.08.012.

Leybaert L. 2005. Neurobarrier coupling in the brain: a partner of neurovascular and neurometabolic coupling? Journal of Cerebral Blood Flow \& Metabolism 25(1):2-16 DOI 10.1038/sj.jcbfm.9600001.

Leybaert L, De Bock M, Van Moorhem M, Decrock E, De Vuyst E. 2007. Neurobarrier coupling in the brain: adjusting glucose entry with demand. Journal of Neuroscience Research 85(15):3213-3220 DOI 10.1002/jnr.21189.

Liddelow SA, Barres BA. 2017. Reactive astrocytes: production, function, and therapeutic potential. Immunity 46(6):957-967 DOI 10.1016/j.immuni.2017.06.006.

Lindauer U, Dirnagl U, Füchtemeier M, Böttiger C, Offenhauser N, Leithner C, Royl G. 2010. Pathophysiological interference with neurovascular coupling-when imaging based on hemoglobin might go blind. Frontiers in Neuroenergetics 2:25 DOI 10.3389/fnene.2010.00025.

Liu S, Cai W, Liu S, Zhang F, Fulham M, Feng D, Pujol S, Kikinis R. 2015. Multimodal neuroimaging computing: a review of the applications in neuropsychiatric disorders. Brain Informatics 2(3):167-180 DOI 10.1007/s40708-015-0019-x.

Maggioni E, Molteni E, Arrigoni F, Zucca C, Reni G, Triulzi FM, Bianchi AM. 2013. Coupling of fMRI and NIRS measurements in the study of negative BOLD response to intermittent photic 
stimulation. In: Proceedings of the Annual International Conference of the IEEE Engineering in Medicine and Biology Society. Piscataway: IEEE, 1378-1381.

Magistretti PJ. 2006. Neuron-glia metabolic coupling and plasticity. Journal of Experimental Biology 209(12):2304-2311 DOI 10.1242/jeb.02208.

Malonek D, Grinvald A. 1996. Interactions between electrical activity and cortical microcirculation revealed by imaging spectroscopy: implications for functional brain mapping. Science 272(5261):551-554 DOI 10.1126/science.272.5261.551.

McCaslin AFH, Chen BR, Radosevich AJ, Cauli B, Hillman EMC. 2011. In vivo 3D morphology of astrocyte-vasculature interactions in the somatosensory cortex: implications for neurovascular coupling. Journal of Cerebral Blood Flow \& Metabolism 31(3):795-806 DOI 10.1038/jcbfm.2010.204.

Miller DI, Halpern DF. 2014. The new science of cognitive sex differences. Trends in Cognitive Sciences 18(1):37-45 DOI 10.1016/j.tics.2013.10.011.

Mishra A. 2017. Binaural blood flow control by astrocytes: listening to synapses and the vasculature. Journal of Physiology 595(6):1885-1902 DOI 10.1113/JP270979.

Mishra A, Reynolds JP, Chen Y, Gourine AV, Rusakov DA, Attwell D. 2016. Astrocytes mediate neurovascular signaling to capillary pericytes but not to arterioles. Nature Neuroscience 19(12):1619-1627 DOI 10.1038/nn.4428.

O'Herron P, Chhatbar PY, Levy M, Shen Z, Schramm AE, Lu Z, Kara P. 2016. Neural correlates of single-vessel haemodynamic responses in vivo. Nature 534(7607):378-382

DOI 10.1038/nature17965.

Okamoto M, Dan H, Shimizu K, Takeo K, Amita T, Oda I, Konishi I, Sakamoto K, Isobe S, Suzuki T, Kohyama K, Dan I. 2004. Multimodal assessment of cortical activation during apple peeling by NIRS and fMRI. NeuroImage 21(4):1275-1288 DOI 10.1016/j.neuroimage.2003.12.003.

Perea G, Araque A. 2005. Glial calcium signaling and neuron-glia communication. Cell Calcium 38(3-4):375-382 DOI 10.1016/j.ceca.2005.06.015.

Perea G, Navarrete M, Araque A. 2009. Tripartite synapses: astrocytes process and control synaptic information. Trends in Neurosciences 32(8):421-431 DOI 10.1016/j.tins.2009.05.001.

Petzold GC, Murthy VN. 2011. Role of astrocytes in neurovascular coupling. Neuron 71(5):782-797 DOI 10.1016/j.neuron.2011.08.009.

Phillips AA, Chan FH, Zheng MMZ, Krassioukov AV, Ainslie PN. 2016. Neurovascular coupling in humans: physiology, methodological advances and clinical implications. Journal of Cerebral Blood Flow \& Metabolism 36(4):647-664 DOI 10.1177/0271678X15617954.

Poldrack RA, Yarkoni T. 2016. From brain maps to cognitive ontologies: informatics and the search for mental structure. Annual Review of Psychology 67(1):587-612

DOI 10.1146/annurev-psych-122414-033729.

Pouratian N, Sheth SA, Martin NA, Toga AW. 2003. Shedding light on brain mapping: advances in human optical imaging. Trends in Neurosciences 26(5):277-282 DOI 10.1016/S0166-2236(03)00070-5.

Raichle ME. 2009. A brief history of human brain mapping. Trends in Neurosciences 32(2):118-126 DOI 10.1016/j.tins.2008.11.001.

Rosenblum WI. 1986. Endothelial dependent relaxation demonstrated in vivo in cerebral arterioles. Stroke 17(3):494-497 DOI 10.1161/01.STR.17.3.494.

Roy C, Sherrington C. 1890. On the regulation of the blood-supply of the brain. Journal of Physiology 11(1-2):85-158 DOI 10.1113/jphysiol.1890.sp000321. 
Sasai S, Homae F, Watanabe H, Sasaki AT, Tanabe HC, Sadato N, Taga G. 2012. A NIRS-fMRI study of resting state network. NeuroImage 63(1):179-193

DOI 10.1016/j.neuroimage.2012.06.011.

Sato H, Yahata N, Funane T, Takizawa R, Katura T, Atsumori H, Nishimura Y, Kinoshita A, Kiguchi M, Koizumi H, Fukuda M, Kasai K. 2013. A NIRS-fMRI investigation of prefrontal cortex activity during a working memory task. NeuroImage 83:158-173 DOI 10.1016/j.neuroimage.2013.06.043.

Scarapicchia V, Brown C, Mayo C, Gawryluk JR. 2017. Functional magnetic resonance imaging and functional near-infrared spectroscopy: insights from combined recording studies.

Frontiers in Human Neuroscience 11:419 DOI 10.3389/fnhum.2017.00419.

Scemes E, Spray DC. 2003. The astrocytic syncytium. Advances in Molecular and Cell Biology 31:165-179 DOI 10.1016/S1569-2558(03)31007-0.

Shoham Y, Perrault R, Brynjolfsson E, Clark J, Manyika J, Niebles JC, Lyons T, Etchemendy J, Bauer Z. 2018. The AI Index 2018 Annual Report. Stanford. Available at http://cdn.aiindex.org/2018/AI\%20Index\%202018\%20Annual\%20Report.pdf.

Sotero RC, Trujillo-Barreto NJ. 2007. Modelling the role of excitatory and inhibitory neuronal activity in the generation of the BOLD signal. NeuroImage 35(1):149-165 DOI 10.1016/j.neuroimage.2006.10.027.

Sotero RC, Trujillo-Barreto NJ. 2008. Biophysical model for integrating neuronal activity, EEG, fMRI and metabolism. NeuroImage 39(1):290-309 DOI 10.1016/j.neuroimage.2007.08.001.

Steinbrink J, Villringer A, Kempf F, Haux D, Boden S, Obrig H. 2006. Illuminating the BOLD signal: combined fMRI-fNIRS studies. Magnetic Resonance Imaging 24(4):495-505 DOI 10.1016/j.mri.2005.12.034.

Strangman G, Culver JP, Thompson JH, Boas DA. 2002. A quantitative comparison of simultaneous BOLD fMRI and NIRS recordings during functional brain activation. NeuroImage 17(2):719-731 DOI 10.1016/S1053-8119(02)91227-9.

Sweeney MD, Ayyadurai S, Zlokovic BV. 2016. Pericytes of the neurovascular unit: key functions and signaling pathways. Nature Neuroscience 19(6):771-783 DOI 10.1038/nn.4288.

Sweeney PW, Walker-Samuel S, Shipley RJ. 2018. Insights into cerebral haemodynamics and oxygenation utilising in vivo mural cell imaging and mathematical modelling. Scientific Reports 8(1):1373 DOI 10.1038/s41598-017-19086-z.

Tachtsidis I, Scholkmann F. 2016. False positives and false negatives in functional near-infrared spectroscopy: issues, challenges, and the way forward. Neurophotonics 3(3):030401.

Toga AW. 2015. Brain mapping: an Encyclopedic reference. First Edition. Vol. 1-3. Waltham: Elsevier.

Tong Y, Frederick BD. 2010. Time lag dependent multimodal processing of concurrent fMRI and near-infrared spectroscopy (NIRS) data suggests a global circulatory origin for low-frequency oscillation signals in human brain. NeuroImage 53(2):553-564

DOI 10.1016/j.neuroimage.2010.06.049.

Toyoda H, Kashikura K, Okada T, Nakashita S, Honda M, Yonekura Y, Kawaguchi H, Maki A, Sadato N. 2008. Source of nonlinearity of the BOLD response revealed by simultaneous fMRI and NIRS. NeuroImage 39(3):997-1013

DOI 10.1016/j.neuroimage.2007.09.053.

Venclove S, Daktariunas A, Ruksenas O. 2015. Functional near-infrared spectroscopy: a continuous wave type based system for human frontal lobe studies. EXCLI Journal 14:1145-1152. 
Von Bartheld CS, Bahney J, Herculano-Houzel S. 2016. The search for true numbers of neurons and glial cells in the human brain: a review of 150 years of cell counting. Journal of Comparative Neurology 524(18):3865-3895 DOI 10.1002/cne.24040.

Von Bernhardi R. 2016. Glial cells in health and disease of the CNS. In: Von Bernhardi R, ed. Vol. 949. Cham: Springer International Publishing, Available at https://link.springer.com/book/ 10.1007\%2F978-3-319-40764-7.

Wager TD, Vazquez A, Hernandez L, Noll DC. 2005. Accounting for nonlinear BOLD effects in fMRI: parameter estimates and a model for prediction in rapid event-related studies. NeuroImage 25(1):206-218 DOI 10.1016/j.neuroimage.2004.11.008.

Walsh V. ed. 2016. New horizons in neurovascular coupling: a bridge between brain circulation and neural plasticity. First edition. Amsterdam: Elsevier. 